\title{
Clinical Significance of the Glasgow Prognostic Score in Patients with Gastrointestinal Stromal Tumors
}

\author{
TAKAAKI ARIGAMI ${ }^{1,2}$, YOSHIKAZU UENOSONO $^{2}$, SUMIYA ISHIGAMI $^{1}$, \\ SHIGEHIRO YANAGITA ${ }^{1}$, KEISHI OKUBO ${ }^{1}$, YASUTO UCHIKADO ${ }^{1}$, YOSHIAKI KITA ${ }^{1}$, \\ SHINICHIRO MORI ${ }^{1}$, HIROSHI KURAHARA ${ }^{1}$, KOSEI MAEMURA ${ }^{1}$ and SHOJI NATSUGOE ${ }^{1,2}$ \\ ${ }^{1}$ Department of Digestive Surgery, Breast and Thyroid Surgery, \\ Kagoshima University Graduate School of Medical and Dental Sciences, Kagoshima, Japan; \\ ${ }^{2}$ Molecular Frontier Surgery, Kagoshima University Graduate School of Medical \\ and Dental Sciences, Kagoshima, Japan
}

\begin{abstract}
Aim: To assess the clinical utility of the Glasgow prognostic score (GPS) as a blood predictor of postoperative recurrence in patients with gastric gastrointestinal stromal tumors (GISTs). Patients and Methods: Twenty-nine patients with gastric GISTs undergoing gastrectomy were retrospectively reviewed. Patients were classified based on GPS criteria as follows: GPS of 2: elevated C-reactive protein $(>1.0 \mathrm{mg} / \mathrm{dl})$ and hypoalbuminemia $(<3.5 \mathrm{~g} / \mathrm{dl}), \mathrm{GPS}$ of 1 : one of these hematological abnormalities, and GPS of 0: neither elevated CRP nor hypoalbuminemia. Results: The National Institutes of Health (NIH) classification for a risk stratification demonstrated that 3 (10.3\%), $15(51.7 \%), 5(17.2 \%)$, and 6 $(20.7 \%)$ patients were at very low, low, intermediate, and high risk, respectively, of disease recurrence. GPS criteria classified 24 (82.8\%), five (17.2\%), and no (0\%) patients into GPS of 0,1 , and 2, respectively. Postoperative recurrence was identified in five patients (17.2\%). Disease recurrence correlated with a risk stratification based on the NIH or GPS classification ( $p=0.004$ and $p=0.024$, respectively). Conclusion: The GPS, as well as NIH classification, is a promising blood predictor of disease recurrence in patients with resectable gastric GISTs.
\end{abstract}

Gastrointestinal stromal tumors (GISTs) are one of the most common mesenchymal tumors in the gastrointestinal tract.

Correspondence to: Takaaki Arigami, MD, Ph.D., Department of Digestive Surgery, Breast and Thyroid Surgery, and Molecular Frontier Surgery, Kagoshima University Graduate School of Medical and Dental Sciences, 8-35-1 Sakuragaoka, Kagoshima 8908520, Japan. Tel: +81 992755361, Fax: +81 992657426, e-mail: arigami@m.kufm.kagoshima-u.ac.jp

Key Words: Glasgow prognostic score, gastrointestinal stromal tumors, disease recurrence, blood predictor.
The incidence of GISTs derived from the stomach ranges between $50 \%$ and $60 \%$ and the number of patients with gastric GISTs is higher than those with GISTs of other organs, such as the small intestine, colorectum, and esophagus (1). Furthermore, the prognosis of patients with GISTs has improved due to recent advances in clinical management, including diagnosis, surgical treatments, and molecular targeted therapy $(1,2)$. A risk assessment for disease recurrence in postoperative patients with resectable GISTs has been established based on clinical practice guidelines indicated by the National Comprehensive Cancer Network and European Society for Medical Oncology (3, 4).

To date, several criteria have been used in the assessment of a risk stratification (5-7). The National Institutes of Health (NIH) classification, proposed in 2002 by Fletcher et al., is based on tumor size and mitotic count (5). On the other hand, Miettinen et al. proposed the Armed Forces Institute of Pathology (AFIP) classification according to tumor size, mitotic index, and tumor site (6). Moreover, Joensuu et al. defined the modified NIH classification based on the presence or absence of tumor rupture (7). These classifications play an important role not only in detecting a high-risk group with disease recurrence, but also selecting patients for adjuvant treatments after surgery (1-4). However, difficulties are associated with preoperatively assessing risk stratification for disease recurrence in patients with GISTs. If it is possible to preoperatively predict risk for disease recurrence using conventional blood examinations, this liquid analysis may be one of the most promising tools for planning strategical treatments for patients with GISTs having potentially malignant behavior.

Recent studies have focused on the clinical utility of the Glasgow prognostic score (GPS), assessed based on C-reactive protein (CRP) and serum albumin, as a predictor of tumor progression and prognosis in patients with several gastrointestinal tract cancer, such as esophageal, gastric, and 
colorectal cancer (8-11). However, the clinical impact of GPS in patients with GISTs has not yet been assessed. Consequently, the purpose of the present study was to investigate GPS, NIH, AFIP, and modified NIH classifications in patients with resectable gastric GISTs. We also analyzed the relationship between GPS and postoperative recurrence in order to assess its clinical utility as a blood predictor of disease recurrence.

\section{Patients and Methods}

Patients. Twenty-nine patients (18 men and 11 women; mean age $=64.5$ years, range $=32-80$ years) with resectable gastric GISTs were retrospectively analyzed in this study. All patients underwent gastrectomy at Kagoshima University Hospital (Kagoshima, Japan) between January 2002 and August 2015. Patients were followed-up every 3-6 months after surgery by regular clinical examinations, such as blood tests and computed tomography at Kagoshima University Hospital. The median follow-up period was 735 days (range $=21-4,875$ days).

This retrospective observational study was approved by the Ethics Committee of Kagoshima University (approval number 28-46).

Classifications for a risk stratification. All patients were categorized for risk of disease recurrence based on three established classification systems: the NIH, AFIP, and modified NIH classifications (5-7).

Assessment of GPS. Blood samples were collected within 2 weeks before surgery. CRP and albumin were measured using a JCA-BM automatic analyzer (JEOL Ltd., Tokyo, Japan).

All patients were categorized into three groups based on GPS criteria as follows: GPS of 2: elevated CRP $(>1.0 \mathrm{mg} / \mathrm{dl})$ and hypoalbuminemia $(<3.5 \mathrm{~g} / \mathrm{dl})$; GPS of 1 : one of these hematological abnormalities; GPS of 0: neither elevated CRP nor hypoalbuminemia (11).

Statistical analysis. Relationships between disease recurrence and GPS or the NIH, AFIP, and modified NIH classifications were assessed using the chi-squared and Fisher's exact tests. Univariate and multivariate logistic regression analyses were used to obtain odds ratios (ORs) and their 95\% confidence intervals (CIs) for assessing preoperative predictors of disease recurrence. All data were statistically analyzed using SAS statistical software (SAS Institute Inc., Cary, NC, USA). A p-value of less than 0.05 was considered significant.

\section{Results}

Clinicopathological characteristics of patients with gastric GISTs. Table I shows the clinicopathological characteristics of 29 patients with gastric GISTs reviewed in the present study. Regarding surgical methods, local resection was performed on 22 patients, proximal gastrectomy on two, and distal gastrectomy on five patients. The R0 resection rate was $100 \%$ (29/29). Five (17.2\%) patients received adjuvant chemotherapy using imatinib mesylate.
Table I. Clinicopathological characteristics of patients with gastrointestinal stromal tumors.

\begin{tabular}{lc}
\hline Factor & Value \\
\hline Gender, n (\%) & \\
$\quad$ Male & $18(62.1)$ \\
Female & $11(37.9)$ \\
Mean age (years) & 64.5 \\
Tumor location, n (\%) & \\
$\quad$ Upper & $12(41.4)$ \\
Middle & $12(41.4)$ \\
Lower & $5(17.2)$ \\
Mean tumor size (mm) & 49.9 \\
Mitotic count, $\mathrm{n}(\%)$ & \\
$<5 / 50$ HPFs & $24(82.8)$ \\
$\geq 5 / 50$ HPFs & $5(17.2)$ \\
Curability, n (\%) & \\
R0 & $29(100.0)$ \\
R1 & $0(0.0)$ \\
Adjuvant chemotherapy (imatinib), n (\%) & \\
Yes & $5(17.2)$ \\
No & $23(79.3)$ \\
Unknown & $1(3.5)$ \\
\hline
\end{tabular}

HPFs, High-power fields.

NIH, AFIP, and modified NIH classifications. The NIH classification demonstrated that $3(10.3 \%), 15(51.7 \%), 5$ $(17.2 \%)$, and $6(20.7 \%)$ patients were at very low, low, intermediate, and high risk, respectively, of disease recurrence. According to the AFIP classification, 4 (13.8\%), $14(48.3 \%), 3(10.3 \%), 5(17.2)$, and $3(10.3 \%)$ patients were at no, very low, low, moderate, and high risk, respectively, of disease recurrence. On the other hand, the modified NIH classification indicated that $4(13.8 \%), 14(48.3 \%), 5$ $(17.2 \%)$, and $6(20.7 \%)$ patients were at very low, low, intermediate, and high risk, respectively, of disease recurrence.

Blood assessment of GPS. The GPS classification system revealed $24(82.8 \%), 5(17.2 \%)$, and no $(0 \%)$ patient with GPS of 0,1 , and 2 , respectively. Table II shows the clinicopathological findings and surgical information in patients with GPS of 1 . In the five patients with GPS of 1 , four $(80.0 \%)$ patients had tumors measuring $\geq 100 \mathrm{~mm}$ or mitotic counts of $\geq 5$ per 50 high-power fields.

Disease recurrence and GPS, NIH, AFIP and modified NIH classifications. Disease recurrence was detected in $5(17.2 \%)$ out of 29 patients. Disease recurrence correlated with a risk stratification based on the NIH, AFIP, modified NIH, and GPS classifications $(p=0.004, p=0.013, p=0.004$, and $p=0.024$, respectively) (Table III). Univariate and multivariate logistic regression analyses identified a promising 
Table II. Clinicopathological findings and surgical information in patients with Glasgow prognostic score of 1.

\begin{tabular}{|c|c|c|c|c|c|c|c|c|c|c|}
\hline Case & $\begin{array}{l}\text { Age (years)/ } \\
\text { gender }\end{array}$ & $\begin{array}{l}\text { Tumor } \\
\text { location }\end{array}$ & $\begin{array}{l}\text { Tumor size } \\
\text { (mm) }\end{array}$ & $\begin{array}{c}\text { Mitotic } \\
\text { count }\end{array}$ & $\begin{array}{c}\text { CRP } \\
(\mathrm{mg} / \mathrm{dl})\end{array}$ & $\begin{array}{l}\text { Albumin } \\
(\mathrm{g} / \mathrm{dl})\end{array}$ & Surgery & Curability & $\begin{array}{c}\text { Adjuvant } \\
\text { chemotherapy }\end{array}$ & Recurrence \\
\hline 1 & 77/Male & M & 25 & $\geq 5 / 50 \mathrm{HPFs}$ & 0.09 & 2.9 & LR & R0 & No & No \\
\hline 2 & 54/Male & M & 42 & $<5 / 50 \mathrm{HPFs}$ & 0.24 & 3.2 & LR & R0 & No & No \\
\hline 3 & 74/Male & $\mathrm{L}$ & 100 & $<5 / 50 \mathrm{HPFs}$ & 0.85 & 3.3 & DG & R0 & Unknown & Liver \\
\hline 4 & 73/Female & $\mathrm{U}$ & 120 & $<5 / 50 \mathrm{HPFs}$ & 1.09 & 3.8 & PG & R0 & Yes & Dissemination \\
\hline 5 & 77/Male & $\mathrm{U}$ & 160 & $\geq 5 / 50 \mathrm{HPFs}$ & 0.17 & 3.3 & LR & R0 & Yes & Dissemination \\
\hline
\end{tabular}

CRP: C-Reactive protein; DG, distal gastrectomy; HPFs, high-power fields; M, middle third of stomach; L, lower third of stomach; LR, local resection; PG, proximal gastrectomy.

preoperative predictor of disease recurrence. Preoperative candidate predictors included gender, age, tumor location, tumor size, and GPS. The univariate logistic regression analysis demonstrated that preoperative larger tumor size and high GPS were significant predictors of disease recurrence $(p=0.006$ and $p=0.013$, respectively) (Table IV). Only preoperative larger tumor size was an independent predictive factor of disease recurrence in the multivariate logistic regression analysis $(p=0.028)$ (Table IV).

\section{Discussion}

We hypothesized that GPS is a promising blood marker for predicting disease recurrence in patients with resectable gastric GISTs. Therefore, we herein assessed the relationship between disease recurrence and GPS, as well as the NIH, AFIP, and modified NIH classifications. To the best of our knowledge, this is the first study to investigate the clinical utility of GPS as a blood predictor of disease recurrence in patients with gastric GISTs.

We initially investigated risk stratification for disease recurrence based on the NIH, AFIP, and modified NIH classifications. The NIH and modified NIH classification systems identified five (17.2\%) and six (20.7\%) patients at an intermediate and high risk, respectively, of disease recurrence. The disease recurrent rate was significantly higher in patients in the intermediate- and high-risk groups than in those in the very low- and low-risk groups $(p=0.004)$. These results indicate a close relationship between disease recurrence and risk stratification by these classification systems. However, a mitotic index is needed to categorize patients based on these risk classification systems. Consequently, it is impossible to preoperatively discriminate patients with a high recurrent risk from those with a low recurrent risk using these systems.

GPS is a representative blood marker associated with systemic inflammatory responses (11). To date, we have demonstrated that the blood assay for the assessment of GPS is a prominent tool to predict tumor progression, prognosis, and therapeutic response to chemotherapy in patients with
Table III. Disease recurrence and Glasgow prognostic score (GPS), National Institutes of Health (NIH), Armed Forces Institute of Pathology (AFIP), and modified NIH classifications.

\begin{tabular}{llrl}
\hline & \multicolumn{2}{c}{ Recurrence } & \\
\cline { 2 - 3 } & \multicolumn{1}{c}{ Yes } & No & p-Value \\
\cline { 2 - 3 } & & & \\
NIH classification & & & 0.004 \\
$\quad$ Very low-low risk & $0(0 \%)$ & $18(75.0 \%)$ & \\
$\quad$ Intermediate-high risk & $5(100 \%)$ & $6(25.0 \%)$ & \\
AFIP classification & & & \multirow{2}{*}{0.013} \\
None-very low-low risk & $1(20.0 \%)$ & $20(83.3 \%)$ & \\
Moderate-high risk & $4(80.0 \%)$ & $4(16.7 \%)$ & \\
Modified NIH classification & & & \\
$\quad$ Very low-low risk & $0(0 \%)$ & $18(75.0 \%)$ & 0.004 \\
Intermediate-high risk & $5(100 \%)$ & $6(25.0 \%)$ & \\
GPS classification & & & \\
0 & $2(40.0 \%)$ & $22(91.7 \%)$ & 0.024 \\
1 & $3(60.0 \%)$ & $2(8.3 \%)$ & \\
2 & $0(0.0 \%)$ & $0(0.0 \%)$ & \\
\hline
\end{tabular}

gastric cancer (12). In this study, we focused on the clinical significance of GPS as a predictor of disease recurrence in patients with resectable gastric GISTs. Disease recurrence was detected in three $(60.0 \%)$ out of five patients with GPS of 1 . However, $22(91.7 \%)$ out of 24 patients with GPS of 0 had no recurrence. These results demonstrate a close relationship between GPS and disease recurrence in patients with resectable gastric GISTs $(p=0.024)$. Furthermore, a univariate logistic regression analysis showed that GPS was a promising preoperative predictor of disease recurrence $(p=0.013)$. Unfortunately, the multivariate logistic regression analysis did not select GPS as an independent predictor of disease recurrence due to the lack of significance $(p=0.063)$. This result in the multivariate logistic regression analysis may have been influenced by the small sample size. Therefore, it may be essential to discriminate patients with GPS of 1 from those with GPS of 0 in the clinical management of resectable gastric GIST. Moreover, preoperative imatinib therapy may be recommended as a strategical treatment for preventing 
Table IV. Logistic regression analyses for disease recurrence.

\begin{tabular}{|c|c|c|c|c|c|c|c|}
\hline \multirow[b]{2}{*}{ Preoperative factor } & & \multicolumn{3}{|c|}{ Univariate analysis } & \multicolumn{3}{|c|}{ Multivariate analysis } \\
\hline & & Odds ratio & $95 \% \mathrm{CI}$ & $p$-Value & Odds ratio & $95 \% \mathrm{CI}$ & $p$-Value \\
\hline Gender & Male/female & 0.9 & $0.125-7.834$ & 0.917 & & & \\
\hline Age & $\geq 65 /<65$ Years & 1.1 & $0.150-9.291$ & 0.945 & & & \\
\hline Tumor location & Upper/middle, lower & 2.5 & $0.350-21.900$ & 0.356 & & & \\
\hline Preoperative tumor size & $>50 / \leq 50 \mathrm{~mm}$ & 20.0 & $2.262-454.091$ & 0.006 & 15.5 & $1.331-469.193$ & 0.028 \\
\hline GPS & $1-2 / 0$ & 16.5 & $1.815-214.805$ & 0.013 & 11.9 & $0.872-325.141$ & 0.063 \\
\hline
\end{tabular}

CI, Confidence interval; GPS, Glasgow prognostic score.

disease recurrence in patients with GPS of 1 . Kubota et al., in a study of 1,017 patients with resectable gastric cancer, reported that GPS was a promising predictor of long-term survival associated with disease recurrence in curable gastric cancer surgery (10). These findings indicate that GPS has potential utility for risk stratification of disease recurrence in patients with gastric GISTs as well as gastric cancer.

This study had several limitations. The median follow-up period was only 735 days. Moreover, the present study was based on a retrospective analysis of a small number of patients $(n=29)$ at a single institution. These limitations may have resulted in biases. However, this was a preliminary study to assess the clinical utility of GPS in patients with gastric GISTs. Accordingly, further large validation studies using patients with resectable gastric GISTs are needed in order to strengthen our conclusion.

In conclusion, we suggest that GPS, as well as the wellknown risk grouping such as the NIH, AFIP, and modified NIH classifications, has a potential as a blood predictor of disease recurrence in patients with resectable gastric GISTs. We may be able to discriminate patients with resectable gastric GISTs having potentially malignant behavior based on liquid analyses using GPS in preoperative management.

\section{Conflicts of interest}

The Authors have no conflicts of interest or financial ties to disclose.

\section{References}

1 Nishida T, Blay JY, Hirota S, Kitagawa Y and Kang YK: The standard diagnosis, treatment, and follow-up of gastrointestinal stromal tumors based on guidelines. Gastric Cancer 19: 3-14, 2016.

2 Liegl-Atzwanger B, Fletcher JA and Fletcher CD: Gastrointestinal stromal tumors. Virchows Arch 456: 111-127, 2010.

3 Demetri GD, von Mehren M, Antonescu CR, DeMatteo RP, Ganjoo KN, Maki RG, Pisters PW, Raut CP, Riedel RF, Schuetze S, Sundar HM, Trent JC and Wayne JD: NCCN Task Force report: update on the management of patients with gastrointestinal stromal tumors. J Natl Compr Canc Netw 8(Suppl 2): S1-S41, 2010.
4 ESMO/European Sarcoma Network Working Group: Gastrointestinal stromal tumours: ESMO clinical practice guidelines for diagnosis, treatment and follow-up. Ann Oncol 25(Suppl 3): iii21-26, 2014.

5 Fletcher CD, Berman JJ, Corless C, Gorstein F, Lasota J, Longley BJ, Miettinen M, O'Leary TJ, Remotti H, Rubin BP, Shmookler B, Sobin LH and Weiss SW: Diagnosis of gastrointestinal stromal tumors: a consensus approach. Hum Pathol 33: 459-465, 2002.

6 Miettinen $\mathrm{M}$ and Lasota J: Gastrointestinal stromal tumors: pathology and prognosis at different sites. Semin Diagn Pathol 23: 70-83, 2006.

7 Joensuu H, Rutkowski P, Nishida T, Steigen SE, Brabec P, Plank L, Nilsson B, Braconi C, Bordoni A, Magnusson MK, Sufliarsky $\mathrm{J}$, Federico M, Jonasson JG, Hostein I, Bringuier P-P and Emile J-F: KIT and PDGFRA mutations and the risk of gastrointestinal stromal tumor recurrence. J Clin Oncol 33: 634-642, 2015.

8 Ishizuka M, Nagata $\mathrm{H}$, Takagi $\mathrm{K}$, Horie $\mathrm{T}$ and Kubota $\mathrm{K}$ : Inflammation-based prognostic score is a novel predictor of postoperative outcome in patients with colorectal cancer. Ann Surg 246: 1047-1051, 2007.

9 Vashist YK, Loos J, Dedow J, Tachezy M, Uzunoglu G, Kutup A, Yekebas EF and Izbicki JR: Glasgow prognostic score is a predictor of perioperative and long-term outcome in patients with only surgically treated esophageal cancer. Ann Surg Oncol 18: 1130-1138, 2011.

10 Kubota T, Hiki N, Nunobe S, Kumagai K, Aikou S, Watanabe R, Sano T and Yamaguchi T: Significance of the inflammationbased Glasgow prognostic score for short- and long-term outcomes after curative resection of gastric cancer. J Gastrointest Surg 16: 2037-2044, 2012.

11 McMillan DC: The systemic inflammation-based Glasgow prognostic score: a decade of experience in patients with cancer. Cancer Treat Rev 39: 534-540, 2013.

12 Arigami T, Uenosono Y, Ishigami S, Okubo K, Kijima T, Yanagita S, Okumura H, Uchikado Y, Kijima Y, Nakajo A, Kurahara H, Kita Y, Mori S, Maemura K and Natsugoe S: A novel scoring system based on fibrinogen and the neutrophil-lymphocyte ratio as a predictor of chemotherapy response and prognosis in patients with advanced gastric cancer. Oncology 90: 186-192, 2016.

Received October 4, 2016

Revised October 26, 2016

Accepted October 31, 2016 